\title{
Association between vegetarian and vegan diets and depression: a systematic review
}

\author{
Rishika Jain $^{1}$, Alexia Degremont ${ }^{1}$, Elena Philippou ${ }^{1,2}$ and Gladys Oluyemisi Latunde-Dada ${ }^{1}$ \\ ${ }^{1}$ Department of Nutrition and Dietetics, King's College London, London, United Kingdom and \\ ${ }^{2}$ Department of Life and Health Sciences, University of Nicosia, Nicosia, Cyprus
}

Depression is a mental health condition, affecting 350 million people globally ${ }^{(1)}$. Recent evidence has found diet to be a novel approach to reduce depressive symptoms ${ }^{(2)}$. This study aimed to systematically review the literature on the associations between vegetarian and/or vegan diets and the risk or symptoms of depression and compare these with that of omnivorous diets.

A systematic search for relevant papers in Medline via PubMed, EMBASE, Web of Science and Cochrane Library databases was implemented using the following Medical Subject Heading (MeSH) and free text terms: "depression" or "depressive disorder" or "depressive disorder, major" or "postpartum depression" or "bipolar depression" and "vegetarian" or "vegetarian diet" or "vegetarianism" or "vegan" or "vegan diet" or "veganism". Studies were included if they examined the association between vegetarian/vegan diets and depression or depressive symptoms in individuals aged 18 or older. Cohort, cross-sectional, and case-control studies, as well as randomized controlled trials (RCT), published from January $1^{\text {st }}, 2000$ to May 31, 2019, were explored. Studies not published in English; investigating lifestyle patterns or other diets rich in fruits and vegetables but not vegetarian/vegan diets specifically; examining individual dietary nutrients or components rather than diet as a whole; investigating quality of life rather than depression specifically; investigating mental health disorders other than depression; animal studies; systematic reviews; meta-analyses; intervention studies that are not RCTs; conference abstracts; case reports; surveys or letters, were excluded. The systematic review followed Preferred Reporting Items for Systematic Reviews and Meta-Analyses (PRISMA) guidelines. Risks of bias within and between studies were assessed using the quality assessment tools of the National Heart, Lung and Blood Institute of the National Institutes of Health for Case-Control Studies and for Observational Cohort and Cross-Sectional Studies.

Out of 861 articles screened, 19 studies met the inclusion criteria. Fifteen of these studies looked at vegetarian diets specifically, 2 looked at vegan diets specifically, and the remaining 2 looked at both vegetarian and vegan diets. Of the 17 studies investigating associations between vegetarian diets and depression, 5 found vegetarian diets to lower rates of depression, 10 studies found it to increase depression/depressive symptoms, and the remaining 2 found no association. Of the 4 studies investigating associations between vegan diets and depression, 2 studies found the diet to be beneficial against depression, whilst the other 2 found it to increase depression. Overall, the findings revealed conflicting evidence on the association between vegetarian/vegan diets and depression. Due to the heterogeneity of these data, meta-analyses were not performed.

Conflicting evidence was revealed in this systematic review: some studies suggested vegetarian diets had higher rates of depression, whereas others suggested this dietary pattern to be beneficial for depression, its' risk and symptoms. Given the heterogeneity of the studies that examined the association between vegetarian/vegan diets and depression, further, sufficiently powered, research is required to investigate any possible associations or effects.

1. Jacka F N, Mykletun A and Berk, M (2012) BMC Med, 10(1), 149.

2. Quirk S E, Williams L J, O’Neil A et al. (2013) BMC Psychiatry, 13(1): 175. 